\title{
Effectiveness, Safety and Applicability of Wii Therapy in Patients with Chronic Obstructive Pulmonary Diseases
}

\author{
Márjorie Y. Meinhardt ${ }^{1}$, Ahlam Hamid ${ }^{1}$, Janaína Schäfer ${ }^{1,2}$, Daniel Fernando Cruz ${ }^{1}$, Lisiane Lisboa Carvalho ${ }^{3}$ and \\ Andréa Lúcia Gonçalves da Silva ${ }^{1,3}$ \\ 1. Department of Physical Education and Health, University of Santa Cruz do Sul,UNISC, Santa Cruz do Sul, Rio Grande do Sul \\ 96815-900, Brazil \\ 2. Scientific Initiation Fellow, UNISC, Santa Cruz do Sul, Rio Grande do Sul 96815-900, Brazil \\ 3. Research Group "Health Rehabilitation and its Interfaces” from UNISC, Santa Cruz do Sul, Rio Grande do Sul 96815-900, Brazil
}

\begin{abstract}
Aim: We investigated the applicability, effectiveness and safety of Wii Therapy to improve trunk flexibility and reduce symptoms in COPD (chronic obstructive pulmonary disease) patients. Methodology: Case reports of 13 COPD patients from the PR (Pulmonary Rehabilitation) program. They performed 10 sessions of Wii therapy, 2 times/week. The patients were randomized into two groups: Group A ( 5 tennis sessions after 5 bowling sessions) and Group B (10 tennis and bowling sessions, both associated in the same sessions). We analyzed the trunk forward flexibility, dyspnea by Modified Borg Scale Dyspnea index, COPD symptoms by COPD Assessment Test - CAT and the psychological responses by Subjective Experiences Scale. Results: After Wii Therapy, we observed a positive evolution in trunk forward-flexibility, especially in Group B (A = 73.57 \pm 11.07 ; $\mathrm{B}=80.83 \pm 19.85$, no significant). Despite the positive developments of symptoms by the Borg Scale-Dyspnea index (A=0 vs. B $=0.5)$ and $\mathrm{CAT}(\mathrm{A}=$ $11.14 \pm 4.01$ vs. $\mathrm{B}=10.33 \pm 2.33$ ), Group A performed better than Group B. The psychological responses observed were positive experiences for both groups. Conclusion: Wii Therapy focuses only on the individual's movement can be used as a complement to PR. This therapy was effective, safe and easy to apply, in addition to being a resource that provides fun for the patient.
\end{abstract}

Key words: COPD; rehabilitation; flexibility; dyspnea; effectiveness.

\section{Introduction}

COPD (chronic obstructive pulmonary disease) is a major cause of morbidity and mortality in countries with different levels of economic development and it is estimated in 2020 to become the third leading cause of death worldwide $[1,2]$. COPD is defined as a preventable and treatable disease, characterized by airflow limitation, resulting from an abnormal inflammatory reaction to inhaled particles (smoking) and associated with comorbidities [2]. The pathology of COPD is multifactorial, often including systemic inflammation and oxidative stress [3,4].

There is evidence that COPD is harmful to respiratory mechanics and peripheral muscles, as the

\footnotetext{
Corresponding author: Andréa Lúcia Gonçalves da Silva, Ph.D., research field: physiotherapy. E-mail: andreag@unisc.br.
}

structural changes in the respiratory muscles result in muscle weakness and airflow limitation, contributing to physical capacity impairment [2]. Thus, it slowly degrades motor function, leading to a decline in health status and resulting in difficulties and limitations in social life [2]. The COPD physiopathological changes tend to worsen with disease progression and trigger limiting symptoms involving a reduction of activities of daily living and quality of life [5].

Since its introduction in hospitals 10 years ago, Wii therapy was incorporated into the arsenal of resources for rehabilitation as an adjunct to conventional physical therapy [6]. Wii Therapy is the use of Nintendo ${ }^{\circledR}$ Wii video game in the rehabilitation of patients suffering from various physical disabilities, which, through the use of a Wii 
remote, is able to capture the movement's performed [7]. All patients, adults or children with some level of understanding, are able to use this device [6]. Although pulmonary rehabilitation for COPD patients are known and well discussed in the physiotherapy setting, little is known about the effectiveness of Wii as a therapeutic modality. Thus, this study investigated the applicability of Wii therapy, its effectiveness in improving trunk flexibility and decreasing symptoms, as well as its therapeutic use safety in a pulmonary rehabilitation program for patients with COPD.

\section{Methods}

Thirteen COPD patients treated by PR (pulmonary rehabilitation) Research Group, according to Global Initiative for chronic obstructive lung disease guidelines-GOLD [2], in southern Brazil, were included in this study. COPD was diagnosed using clinical history, physical examination, and presence of airflow obstruction, defined as a ratio of forced expiratory volume in one second $\left(\mathrm{FEV}_{1}\right)$ to forced vital capacity (FVC) less than $70 \%$ of predicted value [2]. All individuals answered the personal health questionnaire and signed the informed consent before the interview. The study protocol was approved by the Ethics Committee of UNISC, protocol number 3008/11.

COPD patients were randomized into two groups according to the video game used: A and B (Fig. 1). The COPD included in the study were evaluated before, during treatment (at the 5th session) and at the end of Wii therapy sessions. COPD patients with serious heart disease, orthopedic disease and/or cognitive deficits were excluded from this study.

\subsection{Anterior Trunk Flexibility Assessment}

Anterior trunk flexibility was assessed using a fleximeter (Code Research Institute) positioned in the lateral trunk region (below the right armpit) [8]. The patient was in the standing position and performed the inclination of the trunk forward, up to the maximum limit. The results obtained were compared with the Trunk-amplitude Motion Scale [8].

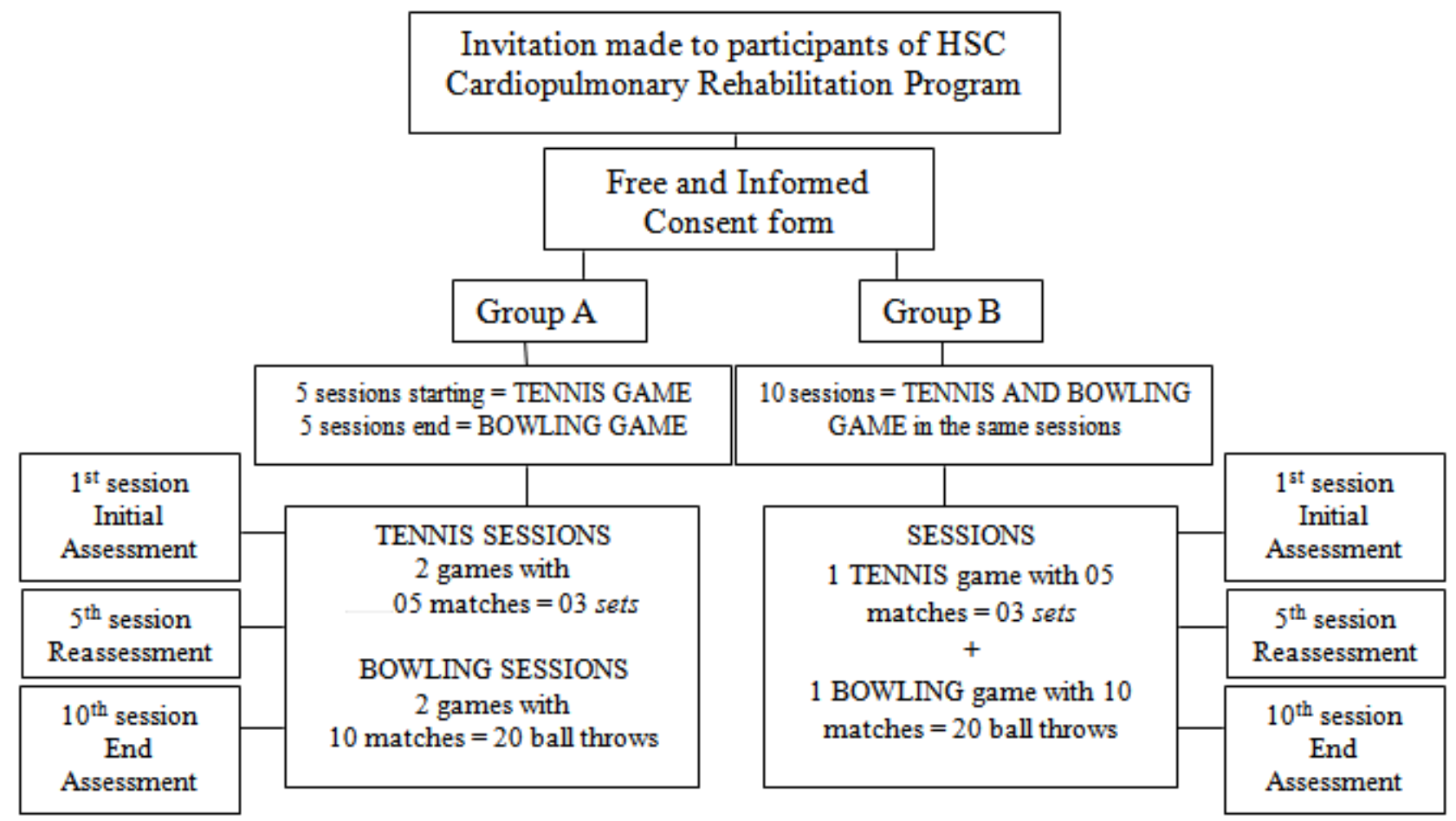

Fig. 1 Methodological phases carried out with the study subjects. 


\subsection{Symptom Assessment}

To evaluate symptoms and their impact on the individuals' lifestyle, the COPD Assessment Test-CAT questionnaire was applied at the beginning of all sessions of Wii therapy. The questionnaire obtains reliable and valid information on the impact of COPD on health status, as well as information on daily symptoms, activity impairment and other disease manifestations [9]. The CAT contains questions resulting in a single final score (minimum 0, maximum 40). Scores $<10$ mean mild symptoms; $10-20=$ moderate, $21-30=$ severe and $>$ $30=$ very severe $[10]$.

We also used the Subjective Experiences Scale to measure the physiological responses resulting from the exercise [11]. The Subjective Exercise Experience Scale is a brief 12-item scale, each item containing a scoring scale of 1-7, which assess the positive well-being, psychological response and fatigue. The results of domain scores allow knowing the feeling of the patient about the experience positive or negative to the individual's health by exercise [12].

\subsection{Nintendo ${ }^{\circledR}$ Wii Exercise Protocol}

The patients underwent 10 sessions of Wii therapy supervised by a physiotherapist, performed 2 times/week for 5 weeks. Nintendo ${ }^{\circledR}$ Wii Sports, which consists in tennis and bowling games (Fig. 1), was used to perform Wii therapy [11]. During the sessions, the participants received voice commands from the physiotherapist about the correct performance of movements and breathing, and all patients used their dominant hand to hold the remote control. After Wii therapy, patients performed muscle stretching arms and legs.

\subsection{Statistical Analysis}

The statistical analyses were performed using the Statistical Package SPSS 18.0 and $P \leq 0.05$ was considered statistically significant. The data are expressed as mean \pm standard deviation. Categorical variables were compared using $\chi^{2}$ tests and numerical variables using Mann-Whitney Test. The comparison among multiple groups was performed by one-way analysis of variance with post-hoc Turkey's multiple comparison test.

\section{Results}

The present study included 18 COPD patients, but throughout the study 5 patients were lost to follow-up and thus, excluded. The patients were randomized into 2 groups, A and B, whose clinical characteristics and smoking history are shown in Table 1. Both groups had similar clinical characteristics. However, those in Group B had a more severe obstructive process in lung function of which cigarette consumption was significantly higher.

For the anterior trunk flexibility, we observed differing clinical outcome during the 10 sessions of Wii therapy (Figs. 2 and 3). The Group B was better than Group A after Wii therapy. Two participants from Group A showed a decrease in anterior trunk flexibility at the end of the treatment, and this decrease occurred when changing the modality of game used, from tennis to bowling, which was not observed in Group B. The results obtained for anterior trunk flexibility, after comparison with the Trunk-amplitude

Group A

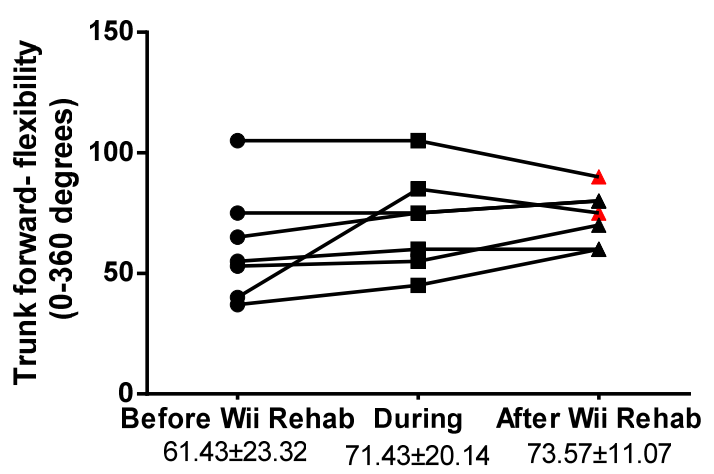

Fig. 2 Anterior trunk flexibility of 7 COPD patients-Group A-measured in degrees before, during (5th session) and after the Wii therapy. The numerical values of the $x$-axis represent the mean \pm standard deviation in degrees of the anterior trunk flexibility, in their 
respective moments of data collection.

\section{Group B}

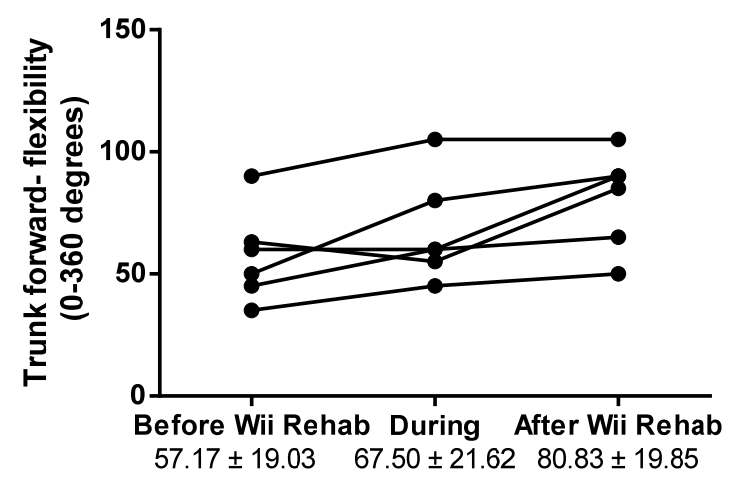

Fig. 3 Anterior trunk flexibility of 6 COPD patients-Group B-n degrees before, during (5th session) and after the Wii therapy. The numerical values of the $\mathrm{x}$-axis represent the mean \pm standard deviation in degrees of the anterior trunk flexibility, in their respective moments of data collection.

Motion Scale [8], were similar for both (Table 2). At the end of Wii therapy, the Groups A and $\mathrm{B}$ remained matched regarding the classification of anterior trunk flexibility and showed improvement.

To verify cardiorespiratory changes resulting from Wii therapy, vital signs were checked in the beginning and ending of each session. The results showed no significant differences between the groups (Table 3 ). The heart rate, respiratory rate, peripheral oxygen saturation remained within the normal range for subjects' age and COPD range. Only systolic blood pressure and diastolic blood pressure improvement at the end sessions.

The results on the degree of dyspnea obtained by the Borg Scale-dyspnea adapted are shown in Fig. 4. The scores for dyspnea remained unchanged during the treatment up to the 5th session and subsequently reduced to zero (i.e., "no breathlessness") at the 10th session of Wii therapy in the Group A. In Group B, the Borg scale showed to be higher in beginning of the session, reducing the final score to "very mild" dyspnea.

The results obtained by CAT test, to assess the effect of Wii therapy on symptom relief, are described in Table 4. Initially, the groups were similar regarding symptom classification in "moderate COPD symptoms". During treatment, Group A showed symptom improvement, maintaining the same score at the end of the Wii therapy. Group B showed symptom worsening during treatment, with improvement at the

Table 1 Clinical characteristics and smoking history of the COPD patients.

\begin{tabular}{|c|c|c|c|}
\hline Characteristics & Group A $(n=7)$ & Group B $(n=6)$ & $P$ value* \\
\hline Age (years) & $64.43 \pm 4.27$ & $61.33 \pm 7.36$ & NS \\
\hline $\operatorname{Sex}(M / F)$ & $3 / 4$ & $4 / 2$ & NS \\
\hline Ethnicity (Caucasian/Non-Caucasian) & $6 / 1$ & $5 / 1$ & NS \\
\hline $\operatorname{BMI}\left(\mathrm{kg} / \mathrm{m}^{2}\right)$ & $25.69 \pm 4.34$ & $24.50 \pm 3.66$ & NS \\
\hline FVC & $66.14 \pm 21.41$ & $51.00 \pm 17.72$ & NS \\
\hline $\mathrm{FEV}_{1}$ & $46.43 \pm 22.81$ & $27.83 \pm 7.27$ & NS \\
\hline $\mathrm{FEV}_{1} / \mathrm{FVC}$ & $66.86 \pm 14.65$ & $58.33 \pm 16.13$ & 0.015 \\
\hline COPD staging (I, II, III, IV)* & $1 / 2 / 3 / 1$ & $0 / 0 / 3 / 3$ & NS \\
\hline Smoking status ${ }^{\#}$ & $0 / 5 / 2$ & $0 / 6 / 0$ & NS \\
\hline Smoking duration $(>30$ years) & 5 & 6 & NS \\
\hline Smoking cessation (years) & $3.50(1-13)$ & $5.42(1-15)$ & NS \\
\hline Cigarettes/year (ex-smokers)* & $7,300(2,190-14,600)$ & $17,640(10,950-21,900)$ & 0.006 \\
\hline Type of cigarette (filtered/non-filtered/others) & $6 / 0 / 1$ & $6 / 0 / 0$ & NS \\
\hline Did any family member have COPD (yes/no/does not know) & $5 / 2 / 0$ & $0 / 5 / 1$ & 0.027 \\
\hline Respiratory disease in childhood (yes/no) & $6 / 0 / 1$ & $1 / 5 / 0$ & NS \\
\hline $\begin{array}{l}\text { Oxygen therapy (yes/no) } \\
\text { Presence of comorbidity (yes/no) }\end{array}$ & $2 / 5$ & $3 / 3$ & NS \\
\hline Degree of dyspnea (mild/moderate/severe/very severe) & $1 / 2 / 3 / 1$ & $3 / 2 / 1 / 0$ & NS \\
\hline Comorbidities (yes/no) & $5 / 2$ & $4 / 2$ & NS \\
\hline
\end{tabular}


Data presented as mean \pm standard deviation and median (minimum-maximum); $* P<0.05$ at Mann-Whitney Test;

${ }^{\#}$ Never/Ex-smoker/Current smoker; NS = non-significant.

Table 2 Anterior trunk flexibility classified according to the “Trunk-amplitude Motion Scale”, by Monteiro, 2000.

\begin{tabular}{lll}
\hline \multirow{2}{*}{ Session } & & Anterior flexibility \\
\cline { 2 - 3 } & Group A & Group B \\
& $n$ / classification & $n$ / classification \\
\hline \multirow{2}{*}{$1^{\mathrm{a}}$} & 4 / "moderately low" & 4 / "moderately low" \\
& 3 / "medium to high" & 2 / "medium to high" \\
$10^{\mathrm{a}}$ & $1 /$ "low" & 1 / "moderately low" \\
& 6 / "medium to high" & 5 / "medium to high" \\
\hline
\end{tabular}

$n=$ number of subjects.

Table 3 Vital signs collected at the beginning and ending of each session in both COPD groups.

\begin{tabular}{|c|c|c|c|c|c|c|c|c|}
\hline \multirow{3}{*}{$\mathrm{N}^{\mathrm{o}}$} & \multicolumn{8}{|c|}{ Groups $A$ and $B$} \\
\hline & \multicolumn{4}{|c|}{ Start of session } & \multicolumn{4}{|c|}{ End of session } \\
\hline & SBP/DBP & HR & $\mathrm{RR}$ & $\mathrm{SpO}_{2}$ & SBP/DBP & $\mathrm{HR}$ & $\mathrm{RR}$ & $\mathrm{SpO}_{2}$ \\
\hline 1 & $\begin{array}{l}122.30 \pm \\
7.25 / 75.38 \pm 6.60\end{array}$ & $79.92 \pm 9.23$ & $23.38 \pm 2.98$ & $\begin{array}{l}93.69 \pm \\
2.68\end{array}$ & $\begin{array}{l}119.23 \pm \\
4.93 / 73.07 \pm 6.30\end{array}$ & $79.69 \pm 8.39$ & $21.30 \pm 3.25$ & $94.07 \pm 2.92$ \\
\hline 2 & $\begin{array}{l}119.23 \pm \\
8.62 / 70.76 \pm 6.40\end{array}$ & $82.61 \pm 9.92$ & $25.23 \pm 4.58$ & $\begin{array}{l}93.84 \pm \\
2.37\end{array}$ & $\begin{array}{l}114.61 \pm \\
5.18 / 71.53 \pm 6.88\end{array}$ & $79.38 \pm 7.89$ & $23.15 \pm 4.72$ & $93.76 \pm 2.31$ \\
\hline 3 & $\begin{array}{l}119.23 \pm \\
7.59 / 74.61 \pm 5.18\end{array}$ & $84.30 \pm 11.03$ & $23.53 \pm 4.40$ & $\begin{array}{l}93.61 \pm \\
2.10\end{array}$ & $\begin{array}{l}116.92 \pm \\
4.80 / 73.07 \pm 6.30\end{array}$ & $83.69 \pm 10.44$ & $22.00 \pm 2.94$ & $93.61 \pm 2.25$ \\
\hline 4 & $\begin{array}{l}117.69 \pm \\
5.99 / 71.53 \pm 8.98\end{array}$ & $84.76 \pm 7.00$ & $23.69 \pm 3.44$ & $\begin{array}{l}93.69 \pm \\
2.62\end{array}$ & $\begin{array}{l}117.69 \pm \\
5.99 / 70.76 \pm 7.59\end{array}$ & $82.23 \pm 6.85$ & $22.30 \pm 5.21$ & $94.00 \pm 2.54$ \\
\hline 5 & $\begin{array}{l}120.76 \pm \\
9.54 / 73.07 \pm 6.30\end{array}$ & $85.15 \pm 9.58$ & $23.00 \pm 3.69$ & $\begin{array}{l}93.76 \pm \\
2.48\end{array}$ & $\begin{array}{l}118.84 \pm \\
9.60 / 77.69 \pm \\
14.23\end{array}$ & $84.92 \pm 7.78$ & $25.46 \pm 12.33$ & $93.84 \pm 2.82$ \\
\hline 6 & $\begin{array}{l}118.46 \pm \\
6.88 / 72.30 \pm 7.25\end{array}$ & $81.92 \pm 9.33$ & $22.46 \pm 3.84$ & $\begin{array}{l}88.38 \pm \\
19.51\end{array}$ & $\begin{array}{l}116.15 \pm \\
5.06 / 73.84 \pm 8.69\end{array}$ & $79.07 \pm 10.09$ & $22.46 \pm 3.28$ & $93.69 \pm 2.35$ \\
\hline 7 & $\begin{array}{l}118.46 \pm \\
8.00 / 73.84 \pm 7.67\end{array}$ & $80.61 \pm 8.23$ & $21.69 \pm 3.03$ & $\begin{array}{l}94.38 \pm \\
2.14\end{array}$ & $\begin{array}{l}117.69 \pm \\
8.32 / 70.76 \pm 8.62\end{array}$ & $82.30 \pm 10.42$ & $22.23 \pm 5.71$ & $94.46 \pm 2.40$ \\
\hline 8 & $\begin{array}{l}121.53 \pm \\
14.63 / 73.07 \pm \\
10.31\end{array}$ & $84.46 \pm 7.91$ & $21.69 \pm 3.03$ & $\begin{array}{l}93.76 \pm \\
2.52\end{array}$ & $\begin{array}{l}120.00 \pm \\
7.07 / 73.07 \pm 6.30\end{array}$ & $80.00 \pm 9.17$ & $22.61 \pm 3.30$ & $94.00 \pm 3.18$ \\
\hline 9 & $\begin{array}{l}120.00 \pm \\
11.54 / 74.61 \pm \\
8.77\end{array}$ & $81.07 \pm 8.53$ & $21.76 \pm 3.78$ & $\begin{array}{l}94.00 \pm \\
2.73\end{array}$ & $\begin{array}{l}116.92 \pm \\
4.80 / 73.84 \pm 6.50\end{array}$ & $79.76 \pm 8.13$ & $21.46 \pm 3.12$ & $93.69 \pm 2.92$ \\
\hline 10 & $\begin{array}{l}122.30 \pm 10.12 / 75 \\
38 \pm 8.77\end{array}$ & $81.69 \pm 9.65$ & $20.46 \pm 4.09$ & $\begin{array}{l}94.84 \pm \\
2.51\end{array}$ & $\begin{array}{l}119.23 \pm \\
6.40 / 73.84 \pm 5.06\end{array}$ & $82.23 \pm 8.41$ & $21.00 \pm 3.91$ & $94.30 \pm 2.62$ \\
\hline
\end{tabular}

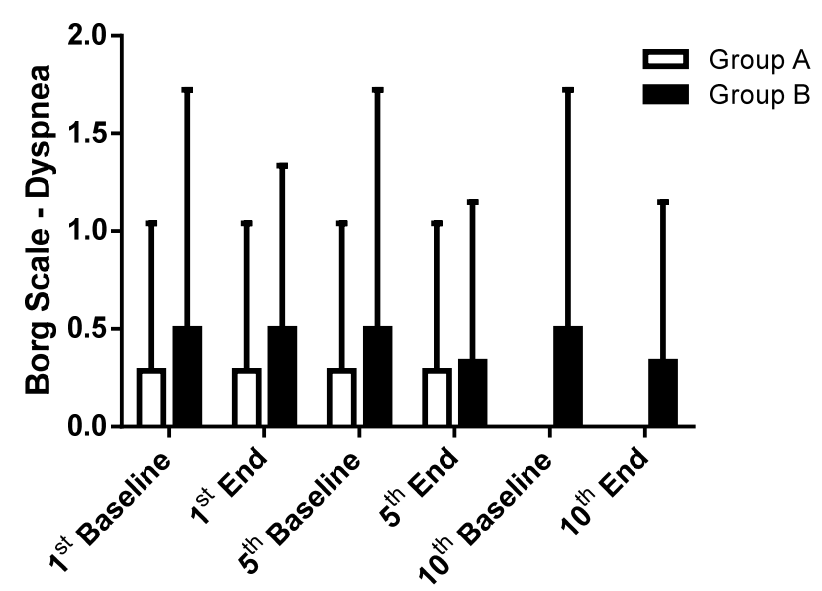

Fig. 4 Borg scale-dyspnea adapted to each patient before and after Wii therapy. Data are presented as mean \pm SD.

end. However, both group still classified as moderate COPD symptoms.

In the Fig. 5 were the results obtained by Subjective Experiences Scale. It was observed that the scoring of positive well-being domain (D1) started similarly in both groups (Group $\mathrm{A}=17.29 \pm 3.63$ vs. Group $\mathrm{B}=$ $17.67 \pm 2.73)$. At the end of treatment, both groups showed evolution, but for Group A (D1 $=20.57 \pm$ 1.61) Wii therapy was a more positive experience than Group B $(\mathrm{D} 1=19.33 \pm 1.86)$. The psychological responses (D2), which involve situations in which the subject feels esteemed or not when performing an 
activity, our results showed improvement compared to the feelings of the patients in both groups $[(1 \mathrm{st}$ session/D2: Group $\mathrm{A}=7.57 \pm 1.90$ vs. Group $\mathrm{B}=$
$7.83 \pm 1.32)$ vs. (10th session/D2: Group $\mathrm{A}=9.29$ \pm 0.95 vs. Group $B=8.67 \pm 1.63)]$, confirming the

Table 4 Results of symptom assessment by CAT in the COPD patients.

\begin{tabular}{llll}
\hline CAT & Group A $(n=7)$ & Group B $(n=7)$ & $P$ value \\
\hline 1st session & $13.14 \pm 6.79$ & $14.83 \pm 4.30$ & NS \\
5th session & $11.71 \pm 3.03$ & $15.33 \pm 4.17$ & NS \\
10th session & $11.14 \pm 4.01$ & $10.33 \pm 2.33$ & NS \\
\hline
\end{tabular}

Data shown as mean \pm standard deviation. $\mathrm{NS}=$ non-significant.

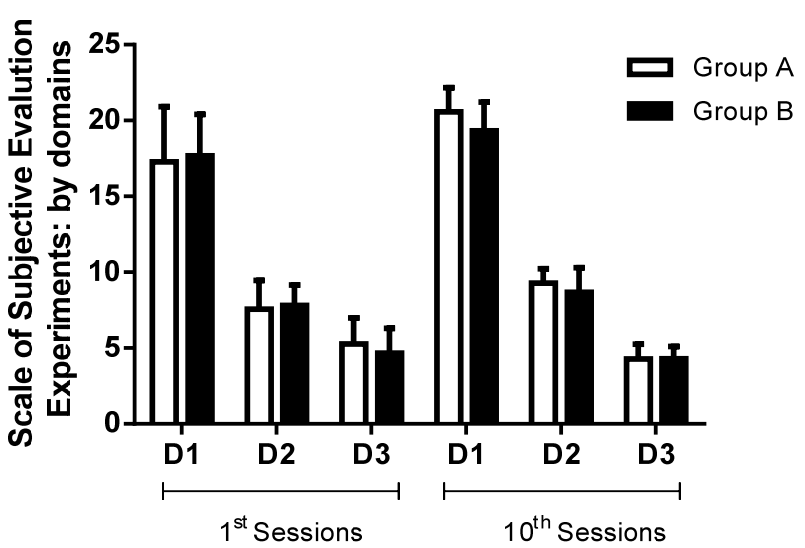

Fig. 5 Total score of the domains that comprise the Subjective Experiences Scale in Groups A and B; D1 = positive well-being; $\mathbf{D 2}=$ psychological response; $\mathrm{D} 3$ = fatigue. Data are expressed as mean \pm SD.

findings of D1 (positive well-being). Regarding the domain fatigue (D3), of which lower score between 4 and 28 reflects less fatigue, the mean score obtained in the 1 st session (Groups: $A=5.29 \pm 1.70, B=4.67 \pm$ 1.63) compared to that obtained at the 10 th session (Groups $\mathrm{A}=4.29 \pm 0.48, \mathrm{~B}=4.33 \pm 0.81$ ) shows a decrease of fatigue for both groups.

\section{Discussion}

The present study investigated the applicability, effectiveness and safety of Wii therapy in improving trunk flexibility and consequent symptom reduction in COPD patients. When analyzing the initial flexibility, positive results were identified, considering that most patients gained flexibility throughout the study. However, even after gaining trunk flexibility, the patients remained classified as having low level of flexibility by Trunk-amplitude Motion Scale [8].
It is noteworthy in both groups the majority of patients had severe obstructive pulmonary process, which leads to pulmonary hyperinflation, deformities and chest rigidity. Chronically, these changes generate remodeling of the inspiratory muscles, especially the diaphragm, which consequently results in structural changes of the chest wall muscles [13]. These alterations associated with structural changes of the spinal column due to the aging process further jeopardize chest mobility. It is known that the movements vary greatly along the column, and due to the inclination of the joint facets, the thoracic spine has great stability with less flexibility [14]. In this respect, one should also consider that the presence of the ribs, in spite of their protective role of vital organs, can become a limiting factor for flexibility [14].

When performing activities with their upper limbs, COPD patients are prone to intense dyspnea, as some muscles of this segment have a dual function, due to their thoracic and extra-thoracic attachment, and, when involved in other activities, they have their respiratory function decreased [15]. When performing the movements proposed in our study, i.e., the Wii therapy game modalities, the subjects made the following movements: when playing tennis they made movements of abduction and adduction of the upper limb, associated with lateral rotation of the trunk; when playing the bowling game, they made movements of hyperextension followed by flexion of the upper limb, associated with flexion and extension of the trunk.

The anterior inclination of the trunk with upper 
limb support seems to be an effective method for relief of dyspnea in COPD patients [13]. The effect of this position does not seem to be related with the severity of airway obstruction, among other factors, but due to increased transdiaphragmatic pressure, a more favorable position of the diaphragm on its force-length curve that significantly improves thorax and abdomen motion [16].

The contraction of the abdominal muscles during expiration lengthens the diaphragm, allowing the muscle to operate close to its optimum length [16]. Moreover, active expiration will increase the elastic recoil pressure of the diaphragm and chest wall and thus, will aid the next inhalation. Furthermore, the slope ahead with the arm support allows the accessory muscles (minor and major pectoral) to significantly contribute to the expansion of the ribcage [16].

Nintendo ${ }^{\circledR}$ Wii has sensors that are sensitive to changes in direction, speed and acceleration [6]. Thus, the subject needs little movement and energy expenditure to perform the movements required by the tennis and bowling modalities, which would not occur in real life [6]. It is a lighter therapy that does not require major efforts, the subjects in our study were able to perform the exercises without having an increase in dyspnea. Both groups showed symptom reduction with a better outcome observed in group B. The subject's dyspnea intensity provides important data on symptom evolution during treatment and this measurement is crucial in clinical practice, allowing physiotherapists to adapt their conduct to achieve therapeutic goals [9].

Vital signs, considered evaluation and cardiorespiratory monitoring parameters, remained stable and within the normal range for patients with COPD in this study, with a small improvement at the end of each session. It is known the cardiorespiratory variables should increase throughout the physical exercise for effective cardiorespiratory fitness to occur $[17,18]$. As it is a milder treatment, which does not require major efforts, the patients were not expected to obtain an increase in the cardiorespiratory parameters and dyspnea degree during the activity. The few changes in these variables may indicate the safety of using this therapeutic modality.

At the end of the our study, the CAT scores revealed an important clinical improvement of symptoms in agreement with the Borg Scale adjusted for dyspnea and psychological responses obtained with the Wii therapy. In addition to routine clinical evaluation, a critical step in patient management is to obtain from the subject, reliable and valid information on the impact of COPD on his/her health [9]. The CAT test should be able to identify specific areas of greater severity to serve as a focal point for the assessment of goals, thereby increasing both the process and outcomes of care [9]. It is suggested that a change in two or more points can indicate clinically significant changes in the subject's health [10].

According to the literature, exercise has a significant impact on the physiological responses in terms of reducing psychological distress and improving positive well-being [11]. The degree to which exercise influence the positive and negative psychological responses can be influenced by the type of activity that is performed. When using the Nintendo ${ }^{\circledR}$ Wii, subjects are attracted by the possibility of playing without having to get accustomed to stranger manual controls [19]. The Wii therapy, in addition to working as a motivational factor for the subjects, might make a difference in the treatment, so the patient seeks the fun to beat his/her own record or even surpass the score of another patient, showing significant improvements in relation to objectives proposed by the service [20]. Social relationships are associated with health outcomes and are therefore extremely important for rehabilitation $[21,22]$.

Used as a single resource, the Wii therapy does not become as effective as conventional pulmonary rehabilitation, which is an indispensable factor for COPD. On the other hand, little change in 
cardiorespiratory variables reinforces the fact that this type of therapy is safe when one wishes only to have an effect on the individual's symptomatology and trunk movement.

It is concluded that the two game modalities employed in the Wii therapy, tennis and bowling, showed improvement in anterior trunk flexibility when applied in an associated manner in the same session. Also, through the Wii therapy, the study COPD patients showed dyspnea improvement as a result from the association of movements performed in the game modalities and the most favorable position of the diaphragm in its tension-length curve. This result was very satisfactory, considering the progression of the disease and its impact on the lifestyle of patients with COPD. The Wii therapy has shown to be a tool that is easy to apply; however, it must be emphasized that its use requires intense monitoring by the physical therapist for patients to perform the correct movements. Based on that, it is believed that the Wii therapy can be used as an adjuvant to conventional rehabilitation programs as it is effective, safe and easy to apply, in addition to being a resource that provides fun for the patient.

\section{Acknowledgments}

We thank the subjects who participated in this study, the CCGS Physiotherapy team and Santa Cruz Hospital.

\section{References}

[1] R. Laniado-Laborín, Smoking and chronic obstructive pulmonary disease (COPD). Parallel epidemics of the 21st century, International Journal of Environmental Research and Public Health (6) (2009) 209-224.

[2] Global Strategy for the Diagnosis, Management and Prevention of COPD. Global Initiative for Chronic Obstructive Lung Disease (GOLD), Update Dec. 2011, http://www.goldcopd.org/uploads/users/files/GOLD_Rep ort_2011_Feb21.pdf (accessed Mar. 6th, 2011).

[3] E.G. Tzortzaki, K. Dimakou, E. Neofytou, K. Tsikritsaki, K. Samara, M. Avgousti, et al., Oxidative DNA damage and somatic mutations, Chest 141 (5) (2012) 1243-1250.

[4] T.L. Verhage, Y.F. Heijdra, J. Molema, L. Daudey, P.R.
Dekhuijzen, J.H. Vercoulen, Adequate patient characterization in COPD: Reasons to go beyond GOLD classification, The Open Respiratory Medicine Journal 3 (2009) 1-9.

[5] E.S. Gladysheva, A. Malhotra, R.L. Owens, Influencing the decline of lung function in COPD: Use of pharmacotherapy, International Journal of COPD (5) (2010) 153-164.

[6] G. Saposnik, R. Teasell, M. Mamdani, J. Hall, W. McIlroy, D. Cheung, et al., Effectiveness of virtual reality using Wii gaming technology in stroke rehabilitation: A pilot randomized clinical trial and proof of principle, Stroke 41 (7) (2010) 1477-1484.

[7] Z. Fritz-Walterf, S. Jones, D. Tjondronegoro, Detecting gesture force peaks for intuitive interaction, in: Proceedings of the 5th Australasian Conference on Interactive Entertainment, Brisbane, Australia, 2008.

[8] G.A. Monteiro, Flexibility Assessment's Manual Fleximeter, in Sanny (Ed.), American Medical Brazil Ltda, São Bernardo do Campo-SP, Brazil, 2005, pp. 6-16.

[9] J.P. Tosato, P.H.F. Caria, Assesment of the muscle activity scoliosis, Rev. Bras. Growth Human Development 19 (1) (2009) 98-102.

[10] P.W. Jones, G. Harding, P. Berry, I. Wiklund, W.H. Chen, N. Kline Leidy, Development and first validation of the COPD assessment test, Eur. Respir. J. 34 (3) (2009) 648-654.

[11] The COPD Assessment Test healthcare professional user guide: Expert guidance on frequently asked questions, http://www.catestonline.org/images/UserGuides/CATHC PUser\%20guideEn.pdf. (accessed Jan. 18, 2011).

[12] E. McAuley, K.S. Courneya, The subjective exercise experiencies scale (SEES): Development and preliminary validation, Journal of Sport \& Exercise Psychology 16 (2) (1994) 163-177.

[13] R.S. Dias, I.L.A. Sampaio, L.S. Taddeo, Physiotherapy vs. Wii: The introduction of the play in the rehabilitation process of patients in physiotherapy, in: VIII Brazilian Symposium on Games and Digital Entertainment Rio de Janeiro, RJ, Brazil, Oct. 8-10, 2009.

[14] A. Ribeiro, J.H.A. Wayhs, M.M. Machado, T.C.M. Fleig, A.L.G. Silva, Analysis of the march in patients with chronic obstructive pulmonary disease, Physiother. Mov. 24 (2) (2011) 211-219.

[15] D. Boaro, A.M.V. Silva, T.C.M. Fleig, A.L.G. Silva, Influence of concentric and eccentric Kabath-exercise on pulmonary inspiratory-function in chronic obstructive pulmonary disease patients, Science Moving Journal 17 (2007) 11-17.

[16] R. Gosselink, Physical therapy in adults with respiratory disorders: Where are we?, Physiotherapy Brazilian Magazine 10 (4) (2006) 361-372. 


\section{Diseases}

[17] J. Carvalho, E. Garmatz, L.L. Carvalho, M.B. Reckziegel, T.C.M. Fleig, A.L.G. Silva, Cardiorrespiratory response to the aerobic excercise with non invasive ventilation at COPD carriers, The FIEP Bulletin 82 (2012) 327-330.

[18] ATS/ACCP Statement on Cardiopulmonary Exercise Testing. Am. J. Respir. Crit. Care. Med. 167 (2) (2003) 211-277.

[19] B. Aguiar, N. Soares, F. Campos, Interface possibilities and new technologies immersion in the game design, in: III Simpósio Nacional ABCiber, São Paulo, SP, Brazil, Nov.16-18, 2009.

[20] M.K. Holden, Virtual environments for motor rehabilitation: Review, Cyber Psychol. Behav. 8 (3) (2005) 187-207.
[21] A. Halding, A. Wahl, K. Heggdal, 'Belonging'. 'Patients' experiences of social relationships during pulmonary rehabilitation, Disability and Rehabilitation 32 (15) (2010) 1272-1280.

[22] L.L. Carvalho, L.K. Dias, C. Classen, G.G. Baldissera, D.F. Cruz, T.C. Fleig, et al., Nintendo Wii therapy in the pulmonary rehabilitation for chronic obstructive pulmonary disease, in: 42nd World Conference on Lung Health of the International Union against Tuberculosis and Lung Disease (The Union), Lille, Franece, 2011. 